Meta

Journal des traducteurs

Translators' Journal

\title{
La traduction et ses dimensions transformatrices
}

\section{Yves Gambier}

Volume 22, numéro 4, décembre 1977

URI : https://id.erudit.org/iderudit/002800ar

DOI : https://doi.org/10.7202/002800ar

Aller au sommaire du numéro

Éditeur(s)

Les Presses de l'Université de Montréal

ISSN

0026-0452 (imprimé)

1492-1421 (numérique)

Découvrir la revue

Citer cet article

Gambier, Y. (1977). La traduction et ses dimensions transformatrices. Meta, 22(4), 252-257. https://doi.org/10.7202/002800ar d'utilisation que vous pouvez consulter en ligne.

https://apropos.erudit.org/fr/usagers/politique-dutilisation/ 


\section{La traduction et ses dimensions transformatrices}

Sur la traduction, il y a un vieux débat, avec de vieilles vérités, de vieilles mesures et autres procédures d'enquête, d'examen-vieilles quant à l'âge qui les a transformées en clichés, en "bon sens », en évidence qu'on ne discute pas, auxquelles on ne revient pas. La traduction est divisée une fois pour toutes? - en méthodes fondamentales. L'une cherche à amener l'auteur vers le lecteur : l'auteur est annexé, il «parle » la langue d'arrivée (LA), plutôt « correcte » puisque écrivain, le sage des normes, sacralisé dans l'ordre social ; et le lecteur n'a «pas l'impression » alors de lire une traduction; cette traduction, toujours aux prises avec son «impossibilité $»$, a engendré des clichés encore en circulation (traducteur-traître ; paradoxes de la traduction ; humilité du traducteur...) et a maintenu l'illusion de la «transparence $^{1}$.

L'autre méthode entreprend de conduire le lecteur vers l'auteur (expressions, style avec des marques de la langue de départ (LD) dans le texte traduit). Ortega, Gasset, Ezra Pound, Ungaretti, Pasternak, Manuel Odorico Mendes... ont servi cette traduction, récréation et dépaysement.

S'agit-il de raviver la querelle de «la $»$ fidélité de la traduction au texte initial ? Mais si cette fidélité - mise sous le boisseau des traditions qui se tiennent bien! - n'était qu'une des formes de l'esprit colonisateur, propre à effacer les différences? Si toute traduction n'était fidèle qu'au prix de simplifications, de réductions, d'édulcorations selon les normes-clichés de la raison, de la langue bourgeoise ? selon le savoir-pouvoir de la classe dite dominante ?

Le traducteur travaille sur un texte en situation (une linguistique de la «parole », une approche analytique du sens ${ }^{2}$ définissent mieux l'opération traduisante qu'une linguistique de la «langue», qu'une stylistique comparée, qu'une grammaire contrastive). D'autre part, la division traditionnelle (traduction littéraire-traduction

1. Cette traduction-métalangage à * 1'humble * service d'un sens premier a permis l'opposition (métaphysique, esthétisante) Écrivain qui ne pouvait qu'être * grand * traducteur, espèce de négrier, d'avorton. Elle explique aussi le dédain des gens de lettres pour les traductions (produits sans prestige qui ne transforment rien), l'absence de celles-ci dans les manuels, les anthologies qui reposent sur l'idéalisation de la littérature.

2. Du sens comme contexte-comportement, réaction à des contraintes linguistiques, à des usages. Du sens comme mémoire de la langue et dynamique de la parole, comme référence et différence. Et non comme vérité des philosophes idéalistes. 
technique) efface, plus qu'elle ne rend compte, l'objectif primordial de vouloirdire, de communication de tout texte ${ }^{3}$. Sur les types de traduction, on relève souvent des jugements qui n'ont rien à voir avec le travail sur les textes; leurs exigences et leurs finalités sont différentes.

Un texte, quel que soit son contenu référentiel, ses informations, accorde faits linguistiques, expressivité, visions subjectives. Un article scientifique n'offre pas une forme neutre ${ }^{4}$ au lecteur ; un montage littéraire ne vit pas seulement pour son auteur, en n'étant qu'un jeu sur le signe et son arbitraire, qu'un jeu sur les signifiants.

La question alors : jusqu'à quand/jusqu'où le texte traduit doit-il censurer ses effets de transformation, faire "comme s'il» n'était pas le produit d'un travail ? Tout travail de création laisse des marques de ses efforts, de ses ratures, de ses manques ; pourquoi pas la traduction - lecture critique et exercice de récréation, à travers les mécanismes, les engrenages d'un texte de départ, vers un autre texte, un milieu-cible?

Jusqu'où un texte en LA peut-il être « lourd 》 (barbarismes, archaïsmes, etc. et autres anomalies, «maladies \$ de langue !) selon les normes du discours dominant? Jusqu'où la contamination entre le texte «parole» de départ et celui «parole » d'arrivée ?

La traduction jusqu'à aujourd'hui a été un effet et un effort de colonisation d'une cuvre sacralisée d'une langue, d'une mentalité... par d'autres, avec complexe de supériorité, un peu (?) comme à l'intérieur d'une aire linguistique, une norme tente de dominer, de corriger, de normaliser les dialectes ; comme la langue bourgeoise tente de récupérer l'argot(?).

La traduction ne consiste-t-elle qu'à décalquer un message (jamais clos, jamais achevé) dans un autre système de signes, déterminé par un contexte différent (historiquement, culturellement, linguistiquement, esthétiquement), c'est-à-dire à l'assimiler, à le digérer, à l'intégrer, à le nationaliser.

Où est et qu'est devenue la traduction à mission d'aide à l'échange international ?

La traduction n'est impossible que pour les linguistes qui la voient au ras des mots. Ne serait-elle qu'une imposture ? toujours inadéquate ? Quel est son rôle ?

Conserver, « enrichir », violer la langue dans laquelle elle apparaît : source d'inventions lexicales, de néologismes, de confrontations, d'échanges... ou occasion et lieu pour définir l'académisme, le purisme (défendre et scléroser) ?

3. Il faut souligner encore le rôle des traductions dans les sources, le développement des littératures nationales, dans l'internationalisation des sciences, dans l'extension des techniques entre les pays inégalement développés, car souvent les théoriciens \& oublient » ce rôle multiforme dans leurs analyses. Certes une histoire générale de la traduction qui ne soit pas seulement événementielle reste à faire.

4. La langue informe ce qu'elle véhicule ; elle est un matériau idéologique qui s'institue socialement comme résultat du processus de communication, sous diverses formes dialectales (sociales, locales, techniques, professionnelles). Cf. F. De Saussure, cours de linguistique générale, 1916 (p. 169) : * la langue est une forme et non une substance... elle est une institution sociale. 
Cette mise en cause de la traduction ne vise pas à des solutions simples/ simplistes; elle cherche à susciter l'installation d'une nouvelle sociologie du traduire et de la traduction; elle cherche à reposer la définition des « qualités » de la traduction, sans en faire un problème de comptabilité de mots, d'unités, sans les réduire à des normes pédagogiques ni à des exercices d'équivalences! Ces «qualités » ont une finalité sociale, remplissent une fonction idéologique. Cette mise en cause enfin s'attache non pas à la complexité de l'opération traduisante mais à la complexité du produit traduit.

Au niveau des structures syntaxiques-mentales, Vinay et Darbelnet ont défini diverses catégories et procédures pour les traductions entre le français et l'anglais ${ }^{5}$ : emprunts, transposition, modulation, équivalence, etc. Le français aurait recours ainsi aux mots-signes, abstraits ; il se situerait au plan de l'entendement. L'anglais davantage orienté vers le plan du réel, du concret et de ses détails se servirait de mots-images, etc.

\section{Exemples : il se sauve au plus profond des taillis he slinks away into the depths of the thicket il vide sa pipe en la tapant contre la table He knocks out his pipe against the table}

De même, A. Malblanc a-t-il essayé d'établir une stylistique comparée du français et de l'allemand ${ }^{6}$, identifiant à la suite d'autres linguistes, stylistique à la langue, confondant structures de langue et analyse de sens d'un texte à traduire. Que le français et l'allemand aient deux modes d'appréhension du réel ne permet pas de formuler une théorie de la traduction!

Ces travaux cités répertorient des procédés, au niveau lexical, phrastique. Mais depuis assez longtemps, depuis Von Humboldt, Whorf..., on savait que les découpages linguistiques ne sont pas les mêmes entre les langues! Or, le traducteur ne peut en rester à ces généralités formelles; il travaille sur un texte où les mots sont actualisés dans une musique, une couleur, où les signes grammaticaux ne se réduisent pas à des morphèmes où les unités ne sont pas isolées les unes des autres, sont des jeux d'ombre d'une voix particulière. Sa question, son obsession, il les rencontre sans cesse : jusqu'où ne pas aller trop loin dans la francisation de l'anglais, la germanisation du français, etc., sans céder à la traduction littérale ni aux tournures idiomatiques figées ?

Même chose au niveau lexical : le traducteur doit-il et jusqu'où inventer des néologismes, emprunter, multiplier les faux-amis... quand sa langue maternelle

5. Jean-Paul Vinay et Jean Darbelnet : stylistique comparée du français et de l'anglais (Montréal - nouvelle édition Beauchemin, 1970; Paris - nouvelle édition Didier, 1976).

6. Didier, 1968 ; où l'auteur confirme que l'allemand reste sur le plan de la sensation, donne une large part au dehors, a un point de vue objectif, est une langue synthétique tandis que le français reste sur le plan de l'entendement, fait une large part au sujet pensant/ au dedans (d'où ses métaphores), a un point de vue subjectif, est une langue analytique. Cf. Mario Wandruszka (essai de linguistique contrastive de l'allemand, de l'anglais, du français, de l'italien, de l'espagnol et du portugais paru en 1969). Cf. Jacques Zajicek, études stylistiques comparatives, néerlandais-français, éd. Mouton-Lahaye, 1973).

7. He swam across the river / il nagea à travers la rivière, phrase possible mais rejetée sans cesse au nom du * bon * français. Cf. Walter Benjamin, dans Mythe et Violence; la tâche du traducteur, éd. Denoël, Paris, 1971. 
ne peut nuancer toutes les «nouveautés » du texte étranger? Doit-il se ranger aux ordres et usages de l'Académie, des dictionnaires ou participer comme tout autochtone à l'évolution de sa langue, tout en gardant à l'esprit que son travail est un travail de communication ${ }^{8}$ ? Jusqu'où ne pas aller trop loin dans l'usage du franglais ${ }^{9}$ ?

Les figures du discours, les systèmes linguistiques sont certes liés à des modèles culturels : celui de LA doit-il (pourquoi ? juqu'où ? selon quels critères ?) « exprimer », « rendre » (avec ses moyens, ses procédés) celui de LD, c.-à-d. effacer les traces de LD-langue et vision ? Le texte à traduire doit-il céder, comme il l'a fait jusqu'à maintenant aux normes de LA, c'est-à-dire satisfaire, combler un type de mentalité, un type de lecture, un type de langue... pour lesquels il n'était pas fabriqué, auxquels il n'était pas destiné ?

L'inverse-LA s'ouvrant, innovant avec $L D$, ne produirait-il qu'un mot à mot, qu'un galimatias... dérangeant pour la paresse intellectuelle/émotive établie du lecteur? Ne serait-il qu'un autre arbitraire ?

La traduction n'agit-elle que pour renverser LD (ex. franciser le grec, l'anglais) en LA ou pour adapter LA à LD (helléniser, angliciser le français...) ?

* Depuis Boileau, la traduction de poésie, en français, n'a été considérée comme possible qu'en vers blancs ou qu'en prose (pour ne pas désordonner les classes poétiques françaises ?) ${ }^{10}$.

* Comment rendre les dialectes des campagnes, des camps du russe en anglais? quel niveau de langue pour quel public qui ignore l'environnement où baigne l'original?

* Comment (re)créer la phraséologie, la terminologie mouvante d'un domaine technique en train de s'élaborer?

* Faut-il rendre un texte ancien (le traducteur n'étant pas un philologue) par un langage hermétique, élitique? La traduction, production idéologique marquée, sera toujours à refaire tandis que le texte en $\mathrm{LD}$ ne vieillirait pas : il se transformerait !

8. Les normes, les limites de réception, de compréhension, d'intelligibilité de tel texte pour tel public resteraient à établir ! Ce qui supposerait mieux connus : l'évaluation des connaissances passives, leur part dans la compréhension d'un message ; le processus de lecture; l'attention auditive ; le rôle et la mesure des redondances, des réactions effets sur les récepteurs du message transmis...

9. Camping, parking... (d'origine française) sont-ils des emprunts à rejeter comme bulldozer, playback... ? Bon matin est-il absurde à priori ? Cf. revue Langages, $\mathrm{n}^{\circ} 28$, décembre 1972, éd. Didier-Larousse : * Le bilinguisme du traducteur », par M. Wandruszka (p. 102-109), sur la malléabilité, la perméabilité réciproque du français, de l'anglais, de l'italien. Dans la même revue, voir les articles de Maurice Pergnier, "Traduction et sociolinguistique * (p. 70-74), et de R. Thieberger, * Le langage de la traduction * (p. 75-84), (notions de milieu-source et de milieu-cible).

10. Quelques cxceptions : c.-à-d. efforts pour adapter, ouvrir la langue-prosodic française au russe avec André Meynieux, Elsa Triolet. Ces exceptions ainsi non seulement soulignent l'aspect * traditionnel $*$ de la traduction mais rappellent le peu d'études françaises sur le rythme, la métrique, la prosodie (comme signifiants). Autres exemples où la traduction remplit sa fonction cognitive-transformatrice : en France, la Renaissance devant l'Antiquité gréco-latine ; le XVIIe face aux mondes italien et espagnol ; le latin transformé par la Bible. 
* La traduction des textes dramatiques doit-elle être une adaptation, concession aux effets du moment, au lieu du spectacle, au public (sociologiquement défini)?

Cette nouvelle perspective (LA contaminée, déformée, détruite en partie par LD) révolution ? peut-elle résoudre les incompatibilités linguistiques qui ont fait les beaux jours des adeptes de la traduction impossible (en débarrassant, en partie, LA de ses normes contraignantes) ? Jusqu'à quel point ?

Faciliterait-elle les recherches en stylistique comparée? Permettrait-elle la constitution d'une poétique comparée sans avoir à attendre une typologie des genres, utopique? Que deviendraient les dissemblances fondamentales entre, par exemple, un texte en russe et un texte en français ?

Cette inversion n'aiderait-elle pas la traduction - ouverture aux inventions à remplir son rôle de vivifier la LA, idiome maternel alors fécondé, contaminé ? et le lecteur à dépasser ce qu'il peut dire - lire, ce qu'il ne peut pas dire - lire, selon les normes rigides d'une société de classes?

Enfin, elle n'exclut pas l'élaboration de théorie(s) de la traduction, mais elle en change les bases, les données : une théorie de la traduction qui reste à faire doit déterminer les objectifs de la traduction et cesser de se chercher un statut propre, une place qui lui permettrait de transcender le savoir et le faire des langues; elle ne peut se borner à une approche linguistique; elle doit s'ouvrir parce que la traduction se fait toujours à partir de textes, messages en situation à « une sémiologie qui n'étudie pas seulement les signes porteurs de signification dans le cadre des structures linguistiques en question mais leur rôle dans l'acte de communication et dans la vie sociale $\gg$. Cette sémiologie devra être autre chose qu'une addition des points de vue, des descriptions de la sociolinguistique, de la psycholinguistique, de la linguistique interne... pour établir, analyser les relations processus dynamiques entre dialectes et langues.

La traduction est un travail de change (transformation de langue et de texte), une partie de plaisir, corrosive des hiérarchies inscrites dans les langues (officielles, autoritaires, totalitaires). Elle est une activité générative qui déterritorialise les langues, contaminent les aires linguistiques/culturelles par leurs à-côtés. Elle ouvre des brèches dans l'espace/les institutions des discours. Elle est le travail incessant, fluctuant des langues-visions, entre elles. Elle est le lieu où texte LD et texte LA se mêlent de ce qui ne les regardait pas jusqu'à maintenant, et ne s'exclut plus, ne se limite plus : non plus changer de textes mais charger l'une des « formes » de l'autre. C'est différent du « respect » plus ou moins docile au texte et à son auteur. Différent du mimétisme, de l'analogie, de la naturalisation du texte, plus ou moins équivalent.

La dichotomie ne se situe plus entre traduction «fidèle » selon des repères verbaux et traduction «libre», ni entre «bonnes » et «mauvaises » traductions qui serait un retour à une critique historiquement datée; la traduction activité de langage, production de texte original désormais se pose les questions suivantes : 
à qui est-elle destinée ? quelle est sa place dans l'ensemble des pratiques d'écriture ? à quoi sert-elle ? qui traduit quoi ?

Jusqu'à quand la traduction servira-t-elle à réduire l'autre au même, à occulter les différences à se faire auxiliaire d'une pensée, d'une démarche dominatrices ?

Dans l'actuelle mise en cause des pratiques sociales que sont la lecture, la science, la littérature, il faut repréciser les finalités sociales de la traduction, son rôle dans les communications culturelles (en dépassant le point de vue comptable, la liste d'auteurs influents...)

Yves Gambier 